Melanie Borchers und Dorothee Graf

\title{
Der E-Bibliotheksschein Anglophone Studien - ein virtueller Lernraum als Kooperationsprojekt zwischen Universitätsbibliothek und einem Fach
}

Zusammenfassung: Der vorliegende Text stellt einen an der Universität DuisburgEssen entwickelten Moodle-Kursraum zur Einführung in die fachwissenschaftliche Literaturrecherche vor. Das Blended-Learning-Projekt wurde gemeinsam von der Fachreferentin der Universitätsbibliothek mit Fachwissenschaftlerinnen des Instituts für Anglophone Studien entwickelt und in den Routinebetrieb überführt. Neben der kooperativen Durchführung ist die curriculare Einbindung des Bibliotheksscheins in die anglistischen Studiengänge eine Besonderheit des Online-Kurses. Konzept, Inhalte und Organisation werden erläutert.

Schlüsselwörter: Informationskompetenz, fachwissenschaftliche Recherche, universitäre Kooperation, curriculare Einbindung/Verankerung

The library competence e-certificate of Anglophone studies - a virtual study space as a co-operation project between the university library and a faculty

\begin{abstract}
The following paper presents a Moodle study space for the introduction to the specialized scientific literature research, developed at the university Duisburg-Essen. The Blended-Learning project was developed and given practical application by the expert of the university library, in co-operation with specialists of the faculty for Anglophone studies. Apart from the co-operative execution, the curricular integration of the library competence certificate into the English studies is a special feature of the online course. Concept, contents und organizational structure are explained below.
\end{abstract}

Keywords: information competence, specialized scientific research, university co-operation, curricular integration

DOI 10.1515/bd-2014-0125

Melanie Borchers: contact@melanieborchers.de

Dorothee Graf: dorothee.graf@uni-due.de 


\section{Einleitung}

Der E-Bibliotheksschein Anglophone Studien ist ein Blended-Learning-Projekt, das gemeinsam von der Fachbibliothek Geistes- und Gesellschaftswissenschaften am Campus Essen und dem Institut für Anglophone Studien an der Universität Duisburg-Essen realisiert wurde. Es handelt sich hierbei um einen Moodle-Kursraum, der Studierenden des Faches und anderen Interessierten propädeutische Kompetenzen zur fachspezifischen Literaturrecherche und Medienkompetenz mit Hilfe digitaler Medien vermittelt.

\section{Der virtuelle Lernraum}

Erste Überlegungen zu diesem Projekt formulierten die damalige Bibliotheksbeauftragte des Faches Anglophone Studien, Melanie Borchers, und die Fachreferentin der Universitätsbibliothek, Dorothee Graf. Zudem beteiligten sich neben einer Hilfskraft für die technische Umsetzung noch die Fachkolleginnen Dr. Stefanie Caeners und Margit Hempel an der Konzeption des Kursraums. Die gemeinsam von Caeners und Graf durchgeführte Blockveranstaltung How to write a term paper stand Pate bei der inhaltlichen Entwicklung.

Ausgangspunkte für den E-Bibliotheksschein waren zwei existierende Angebote der Universitätsbibliothek: Bereits seit 2005 wird der Besuch von drei aufeinander aufbauenden Schulungsveranstaltungen $\mathrm{zu}$ Bibliothek und allgemeiner und fachlicher Literaturrecherche mit dem sogenannten Bibliotheksschein kreditiert (vgl. www.uni-due.de/ub/abisz/schein.shtml). Hier kooperiert die Informationsabteilung mit den einzelnen Fachreferenten, denn während die ersten beiden, allgemein gehaltenen Schulungen (,Einführung in die Nutzung der Bibliothek“ und „Allgemeine Literaturrecherche und Fernleihe“) aus dem gestuften Konzept von Kolleginnen der Information übernommen werden, wird für den dritten Teil („Fachbezogene Literaturrecherche“) der Inhalt fachlich differenziert und eine fachwissenschaftlich bezogene Datenbankeinführung durch den jeweiligen Fachreferenten abgehalten. Mit dem Bibliotheksschein wird folglich eine fachbezogene Informationskompetenz bescheinigt. 


\section{UNIVERSITÁT \\ DEU'S ISBN R G}

Offen im Denken

Ub $\mid \begin{gathered}\text { universitäts } \\ \text { bibliothek }\end{gathered}$

\section{Bibliotheksschein}

Frau

Matrikelnr.

hat am dreistufigen Veranstaltungsprogramm (6 UE) der Universitätsbibliothek Duisburg-Essen, zum Erwerb von

Informationskompetenz

im Fach teilgenommen.

Die Schulungsreihe vermittelt folgende fachbezogene und übertragbare Basiskompetenzen:

- Kenntnis von Informationsquellen und -typen:

- Lokale und überregionale Bibliothekskataloge

- Bibliografische und fachspezifische Datenbanken

- Wissenschaftliche Internetportale

- Kritische Bewertung der Informationen

- Entwicklung effektiver Recherchestrategien

Der Bibliotheksdirektor

Essen, den

Abb. 1: Formular des Bibliotheksscheins der Universitätsbibliothek Duisburg-Essen. 
Der zweite Ausgangspunkt war das nach einigen Jahren eingeführte Online-Angebot für die bislang ausschließlich in Präsenzlehre durchgeführten ersten beiden allgemeinen Schulungen. Die Universitätsbibliothek stellt hierzu auf der in der gesamten Universität genutzten Plattform Moodle zwei Kursräume bereit, die Übungsmaterialien, Links, Lektionen und einen Abschlusstest enthalten und für alle Universitätsangehörige frei und kostenlos nutzbar sind.

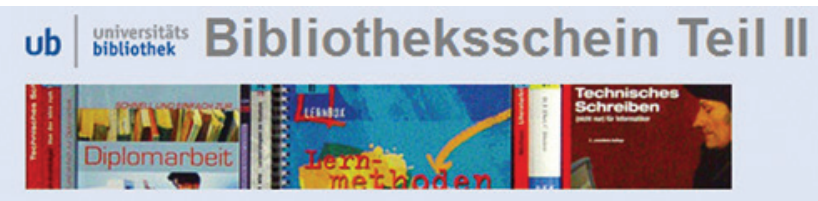

\section{Allgemeine Literaturrecherche und Fernleihe}

Abb. 2: Der zweite der aufeinander aufbauenden Online-Kurse zum Bibliotheksschein (Grafik: Beate Baurmann).

Während die fachliche Literaturrecherche bis dato nur durch Präsenzveranstaltungen im Schulungsraum der Bibliothek abgehalten wurde, haben sich die beiden Moodle-Kurse zur Einführung in die UB sowie zur Literaturrecherche und Fernleihe als Ergänzung und zunehmend als Ersatz für die Präsenzschulungen entwickelt. Gefördert wurde diese Entwicklung durch die 2012 eingeführte Verpflichtung, die Tests zu absolvieren und zu bestehen, mit denen das OnlineAngebot in Moodle abgeschlossen wird. Mit dem Projekt E-Bibliotheksschein Anglophone Studien sollte erstmalig ein Online-Kurs auch für die fachbezogene Literaturrecherche entwickelt und damit der komplett onlinegestützte Erwerb des Bibliotheksscheins ermöglicht werden.

Parallel zur technischen Grundlage standen die curriculare Einbindung und damit die verpflichtende Teilnahme in den anglistischen Studiengängen im Fokus der Kooperation. Hier erwies sich das gestufte Konzept als hilfreich, denn die einzelnen Module des Bibliotheksscheins können so „zu verschiedenen Zeitpunkten während der ersten drei Studiensemester im Fach Anglophone Studien belegt werden. Alle Studierenden des Faches werden auf diese Art unabhängig vom gewählten Abschluss bzw. Studiengang adäquat in die allgemeine und die fachbezogene Literaturrecherche eingeführt. Durch die jeweiligen Modulprüfungen kommen die propädeutischen Kenntnisse auch sofort am konkreten Beispiel zur Anwendung“ (vgl. https://www.uni-due.de/ub/schulung/anglistik_eschein\#curriculum). 


\section{Von der Vermittlung der Inhalte zum Abschlusstest}

Der in Moodle 2 konzipierte Kursraum E-Bibliotheksschein Anglophone Studien ist als Ersatz für die ehemals in Präsenzlehre durchgeführte Veranstaltung geplant und behandelt wie diese fünf gleichwertige Informationsbausteine: Strategien der Literaturrecherche, Nachschlagewerke, Datenbanken, Katalog \& Bibliographie und Zitieren. Jeder dieser Bausteine wird in einem Kapitel präsentiert, das Lehr- und Informationsmaterial in verschiedenen medialen Formen enthält und mit einem Test abgeschlossen wird. Als Beispiel sei Kapitel 1 gezeigt:

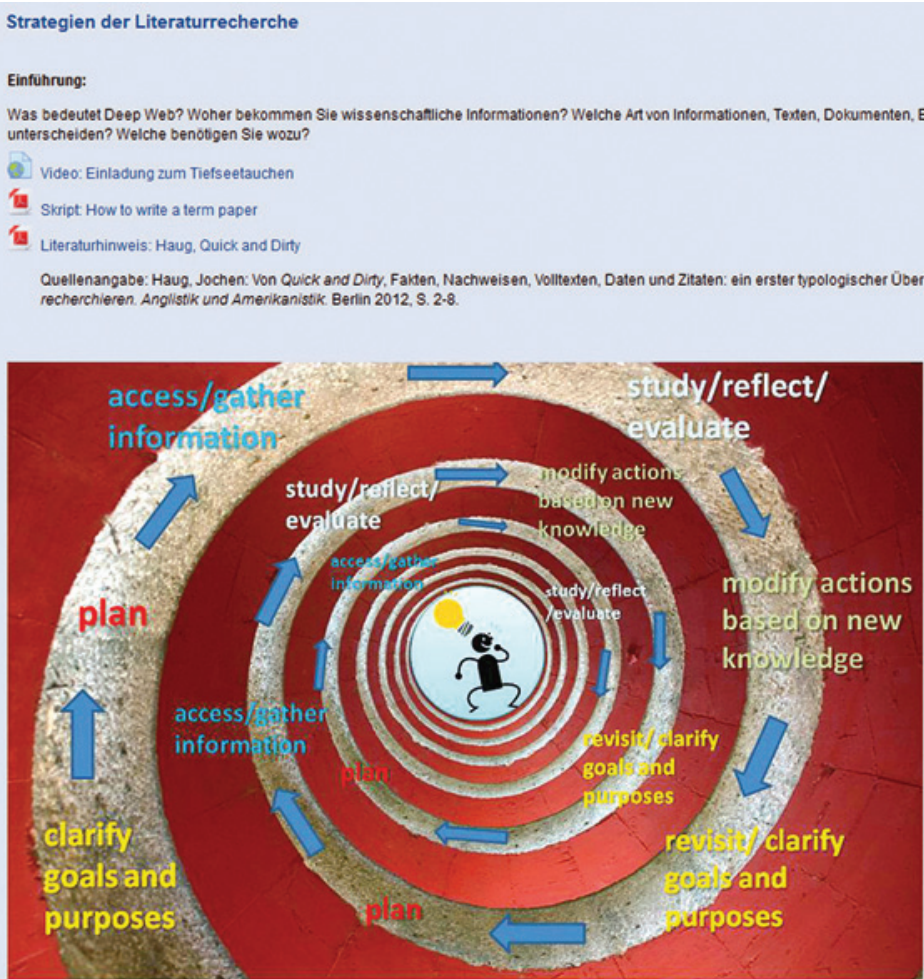

Abb. 3: Das erste Kapitel im Moodle-Kursraum „Fachwissenschaftliche Literaturrecherche: Nachschlagewerke und Datenbanken aus dem Bereich der Anglophonen Studien“ (Grafik: Stefanie Caeners).

Der Aufbau und der dadurch vorgeschlagene Bearbeitungsablauf werden im Folgenden etwas näher vorgestellt. 


\subsection{Fachspezifische Literaturrecherche: Materialien und praktische Übungen}

Im Gegensatz zur Lernpfadsteuerung, die uns mit Moodle 2 zur Verfügung steht und die Wenn-Dann-Bedingungen an Lernfortschritte knüpft, haben wir uns hier für ein alternatives Verfahren entschieden, das den Studierenden die Möglichkeit gibt, sowohl neue Inhalte zu erfahren als auch Übungsaufgaben mit der Lernaktivität Test zu bearbeiten. So misst der E-Bibliotheksschein Anglophone Studien mit der Dozentin, die eher als Lernberaterin oder Coach fungiert, dem generellen didaktischen Ziel des autonomen Lernens einen besonderen Stellenwert bei.

Mit Hilfe von Arbeitsmaterialien werden in einem ersten Schritt die oben erwähnten fünf Informationsbausteine vermittelt. Diese Informationsseiten bieten in Form von Skripten, Textausschnitten oder Videos eine Einführung in die jeweiligen Inhalte für alle, die ihr Wissen auffrischen möchten oder aber zum ersten Mal mit der fachspezifischen Literaturrecherche zu tun haben. Zusätzliches Material, das von der Durchlässigkeit des virtuellen Lernraums profitiert (z. B. externe Links, die auf Seiten mit Spezialwissen verweisen), vervollständigt dieses Informationsangebot. Zwar sind diese fünf Bausteine auf der Hauptseite des Kursraums optisch klar in verschiedenen Kapiteln strukturiert, doch sind sie weder chronologisch noch zeitlich gesteuert und erlauben somit eine Beschäftigung mit der Materie, die den individuellen Bedürfnissen der Studierenden angepasst ist. Um der Binnendifferenzierung des studentischen Vorwissens noch weiter gerecht zu werden, ist diese Lernaktivität optional und nicht verpflichtend für den nächsten Schritt.

Der zweite Schritt hingegen ist verpflichtend. Dieser Schritt, der als Lernaktivität Test umgesetzt wurde, ermöglicht eine Selbstkontrolle über das Vorwissen bzw. die neu vermittelten Inhalte aus den eben erläuterten Arbeitsmaterialien. Studierende erfahren also bereits in dieser Aktivität, die inhaltlich vergleichbar mit der Moodle-Lernaktivität Lektion und methodisch-didaktisch als Übung anzusehen ist, wo ihre Stärken und Defizite liegen, sodass sie gegebenenfalls auf das Informationsangebot zurückgreifen können, bevor sie den Test wiederholen. Wohingegen bei einem Lernpfad das Arbeitsmaterial oft nur bei Stagnation des Lernprozesses offeriert wird, steht es in unserem Kursraum jedem Einzelnen jederzeit zur Verfügung. Somit ist die Wissensvermittlung weniger kontrolliert, aber auch weniger restringiert.

Um ein gutes Zusammenspiel zwischen den Übungsaufgaben und der Lektion zu ermöglichen, werden viele Fragen bzw. Antworten mit einer Rückmeldung kommentiert und verweisen auf das Arbeitsmaterial der jeweiligen Kapitel. Zudem ist weder die Reihenfolge der einzelnen Lernaktivitäten als Tests festgelegt, noch ist die Anzahl der erlaubten Versuche bei diesen begrenzt. Allerdings 
werden für jeden falschen Versuch jeweils $20 \%$ der Punktezahl abgezogen und der Abschlusstest wird erst freigegeben, wenn für jeden der fünf inhaltlich unterschiedlichen Bausteine mindestens $50 \%$ erreicht wurden. Diese automatische Freischaltung lässt sich mit den Bordmitteln der Lernaktivität Test in Moodle realisieren.

\subsection{Lernaktivität Test als abschließender Test für den Nachweis als Studienleistung}

Ist erst einmal der Abschlusstest durch die inhaltliche Vorarbeit und die Erfüllung der vorgeschriebenen Leistungen freigeschaltet, werden den Studierenden zwölf gleich gewichtete Zufallsfragen aus dem Gesamtpool aller Fragen präsentiert. Dafür wurden die Fragen in einer dem Kapitelaufbau entsprechenden hierarchischen Struktur im Fragenpool abgelegt. So ist sichergestellt, dass $\mathrm{zu}$ allen von den Studierenden bereits bearbeiteten und von den Lehrenden für wichtig erachteten Themen auch im Abschlusstest Fragen auftauchen. Das wichtigste Kapitel der Datenbankrecherche ist auch mit der größten Anzahl an Fragen vertreten. Zudem haben wir uns dazu entschlossen, im Abschlusstest die Reihenfolge der Kapitel festzulegen. Diese Festlegung ermöglicht eine bessere Vergleichbarkeit aller Abschlusstests. Durch die von Moodle per Zufall ausgewählten Fragen kann es passieren, dass Studierende im Abschlusstest dieselbe Frage wie im Test des zugehörigen Kapitels erhalten, durch die hohe Zahl an gleich strukturierten, aber inhaltlich verschiedenen Fragen ist dies jedoch unwahrscheinlich und die Antworten bleiben gleichwertig. Insgesamt sind im ständig erweiterbaren Fragenpool derzeit über 100 Fragen, die in Untergruppen mit manchmal nur zwei, manchmal acht oder mehr Fragen aufgeteilt sind. 


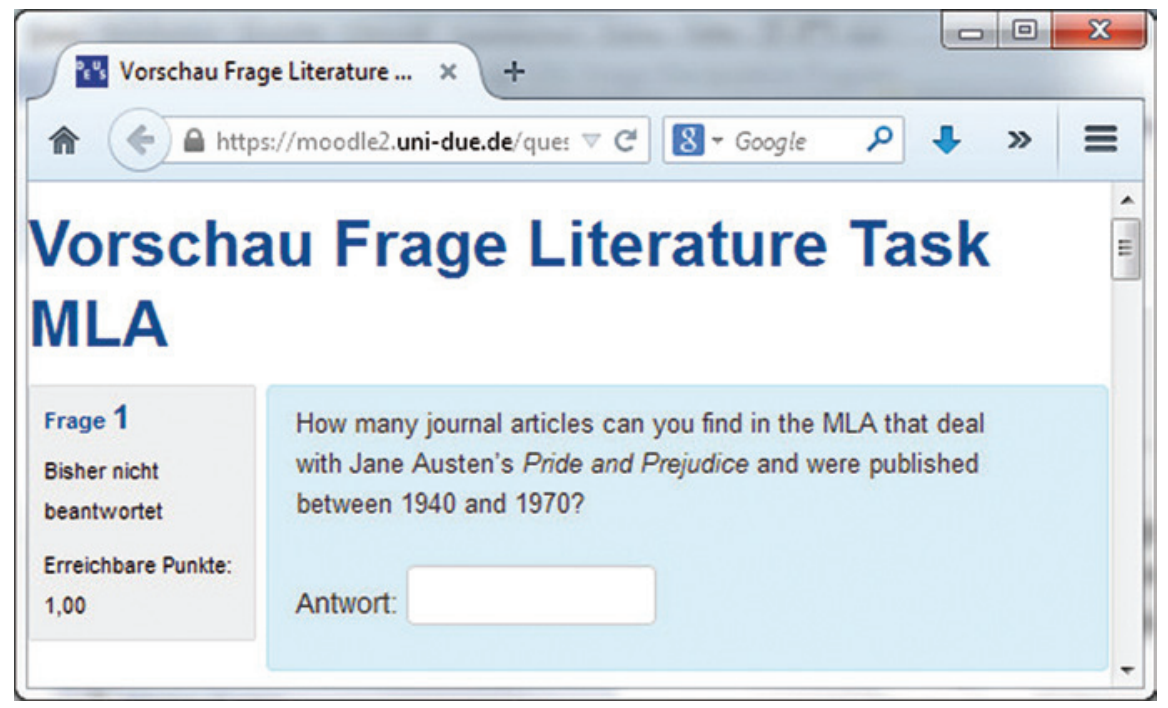

Abb. 4: Beispielaufgabe aus dem Kapitel Exkurs: Katalog und Bibliographie.

Um die Aufgaben wie bei den Vortests automatisch auswerten lassen zu können, beinhaltet der Fragenkatalog ausschließlich Multiple-Choice-Aufgaben oder aber Wahr-Falsch-, Zuordnungs- und Numerische Antwort-Aufgaben sowie Kurzantwort-Aufgaben, deren eindeutige Antwort sich aus der Frage ergibt. Die automatische Auswertbarkeit zieht neben der häufigen Kritik an sogenannten AntwortWahl-Verfahren auch eine entscheidende Konsequenz nach sich, die nicht vom Fach motiviert ist. Aufgrund der Allgemeinen Prüfungsordnung (§ 26 Bewertung der Prüfungsleistungen und Bildung der Prüfungsnoten), wonach bei AntwortWahl-Verfahren ansonsten relative Bestehensgrenzen kalkuliert werden müssen, liegt die Bestehensgrenze für diese Studienleistung der Einfachheit halber ebenfalls bei $50 \%$.

Trotz dieser Einschränkung auf bestimmte Fragentypen und der Notwendigkeit, sie maschinell auswerten zu können, wurden die Aufgaben nicht nur so realitätsgetreu wie möglich gestaltet, es wurde auch sehr viel Wert auf wissenschaftlich anspruchsvolle Fragestellungen gelegt, die für alle Teilfachbereiche relevantes Spezialwissen abfragen (vgl. z. B. die Inhalte zu literaturwissenschaftlichen und linguistischen Datenbanken). Einzelne Fragen sind auch so gestaltet, dass sie nur in den Räumen der Bibliothek bzw. mit Ansicht von Medien vor Ort beantwortet werden können. Da dies nicht nur praktische Probleme birgt (das benötigte Buch könnte ausgeliehen oder verstellt sein) und da erfahrungsgemäß zahlreiche Studierende den Online-Kurs von zuhause aus oder zu Schließungszeiten der Bibliothek absolvieren, wurde die Zahl dieser Fragen reduziert. 
Der Abschlusstest kann zeitlich begrenzt freigeschaltet werden, da er zum einen an Semestertermine und die damit zusammenhängenden Verbuchungsfristen bei Prüfungsämtern gebunden ist, zum anderen gibt eine Pause zwischen Freischaltterminen aber auch die Möglichkeit, Veränderungen am Kursraum vorzunehmen. Dazu zählt neben der Erweiterung des Fragenkatalogs auch die Tatsache, dass gewährleistet sein muss, dass der Kursraum den Neuerungen im Fach und in der Bibliothek (z. B. Einsatz eines neuen Katalogs) angepasst werden kann.

\section{Vorteile gegenüber der eigenständigen UB-Veranstaltung „Fachbezogene Literatur- recherche“ in Präsenzlehre}

Das kooperative Projekt zeichnet sich durch zahlreiche Vorteile aus: Die Zusammenarbeit zwischen Bibliothek und Institut erleichtert die Arbeit für die Fachreferentin und die fachwissenschaftlichen Dozentinnen und erzielt damit Synergieeffekte, nicht zuletzt beim wirtschaftlichen Mitteleinsatz: So wurde eine Hilfskraft durch das Fach bezahlt, die nötige Software aber durch die Universität bzw. die Bibliothek bereitgestellt. Der Charakter als virtueller didaktischer Lernraum mit automatisierter Leistungsüberprüfung hat den besonders in großen Fächern geschätzten Vorteil, große Studierendenzahlen bewältigen zu können (770 Erstsemester allein bei Ersteinsatz des virtuellen Lernraums im Wintersemester 2012/13). Im Fach ist durch den Wegfall der zeit- und planungsintensiven Präsenzveranstaltungen eine Fokussierung auf Seminarinhalte möglich, das Thema Literaturrecherche wird nicht ausgeklammert, aber aus dem fachwissenschaftlichen Seminargeschehen herausgenommen und an die Bibliothek delegiert.

Der E-Bibliotheksschein Anglophone Studien ist als verpflichtende Studienleistung in alle neuen Studienpläne (vgl. http://www.uni-due.de/ub/schulung/ anglistik_e-schein) und damit curricular eingebunden. So erhöht sich der Stellenwert der Bibliotheksrecherche im Studium. Alle Studierenden werden verpflichtend in die fachbezogene Literaturrecherche eingeführt - diese Fertigkeit benötigen sie für die Anfertigung der ersten Hausarbeiten. Wie langjährige bibliothekarische Erfahrung zeigt, führen freiwillige Angebote häufig nicht zu den (vom Fach und der Bibliothek) erwünschten Teilnehmerquoten. Die schrittweise curriculare Einbindung der einzelnen Bestandteile des Bibliotheksscheins erleichterte die Einbettung des kompletten Bibliotheksscheins als verpflichtende Seminarteilleistung und bewirkte eine deutliche Steigerung in den Teilnehmer- 
zahlen: Wurden in den Vorjahren jeweils keine 100 Teilnehmer erreicht, absolvierten im ersten komplett nach dem Kooperationsmodell organisierten Jahr 2013 bereits 329 Studierende den Abschlusstest.

Auch in der Bibliothek führte die Einführung des gemeinsamen MoodleKursraums zur Entlastung von Routineaufgaben. So wurden die organisatorischen und infrastrukturellen Aufgaben zugunsten individueller fachlicher Beratungen reduziert. Insbesondere der von den Studierenden selbst auszudruckende Nachweis über den bestandenen Test reduziert den Bearbeitungsaufwand durch UB-Kolleginnen. Deutlich hervorzuheben ist auch, dass aufgrund des OnlineAngebots der Schulungsraum der Bibliothek wieder mehr für freies Üben und Recherchieren zur Verfügung steht. Nicht zuletzt entsteht durch das Wegfallen der Präsenz-Lehrveranstaltung eine Entlastung sowohl für die Fachreferentin wie für die Studierenden: Die zwar stark standardisierte, aber durch mindestens zehn Termine pro Semester zeitlich aufwändige Veranstaltung muss nicht terminiert und durchgeführt werden und die Studierenden sind in ihren engen Stundenplänen nicht zu einem weiteren Termin vor Ort verpflichtet (von den Schwierigkeiten der Organisation eines solchen Pflichttermins abgesehen).

Insgesamt profitieren vor allem die Studierenden von den Vorteilen der Implementierung des E-Bibliotheksscheins Anglophone Studien. Sie können jederzeit barrierefrei online auf die Moodle-Plattform zugreifen und sind somit orts- und zeitunabhängig. Dies ist sowohl für Menschen mit Behinderungen wie auch für Teilzeitstudierende und Studierende in Elternzeit von Vorteil, weil sie z. B. zuhause diese Studienleistung erbringen können. Die zeitliche Flexibilität wissen alle Studierenden zu schätzen. Über $20 \%$ der Studierenden des zweiten Jahrgangs entschieden sich z.B. dafür, den E-Bibliotheksschein Anglophone Studien an Wochenenden oder Feiertagen zu machen. Wie die Auswertung der Abschlusszeiten des Moodle-Abschlusstests zeigt, wird gerade dieser Aspekt der freien Zeiteinteilung stark genutzt: Fast die Hälfte aller Versuche des Abschlusstests wurden außerhalb regulärer Universitätszeiten absolviert. 


\section{Wann wurde der Abschlusstest beendet? (alle Versuche aus 2013, n=548)}

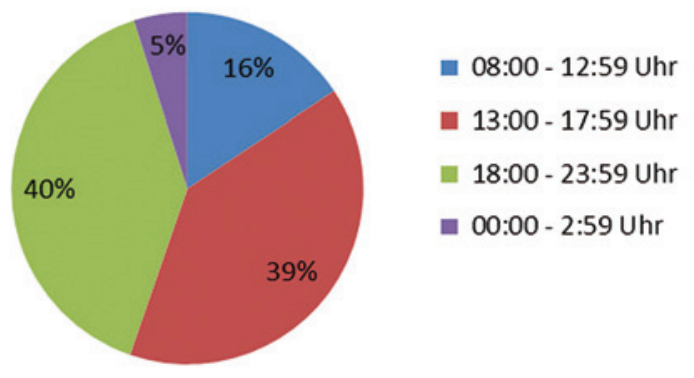

Abb. 5: Auswertung aller Testversuche in 2013 nach Uhrzeit der Beendigung.

Somit hat ein großer Teil der Studierenden die Zeiten selbstbestimmten Lernens den eigenen Bedürfnissen und Möglichkeiten angepasst. $\mathrm{Zu}$ den so fast rund um die Uhr entstandenen Arbeitszeiten wurden vormals nie Präsenzveranstaltungen angeboten.

Auch im Hinblick auf die unterschiedliche Medienkompetenz der Studierenden ist der E-Bibliotheksschein Anglophone Studien barrierefrei. Der Kursraum vermittelt neben den fachlich relevanten Schlüsselkompetenzen, die - ganz nebenbei bemerkt - wegen der Datenbankrecherche am (eigenen) Computer auch einen gesteigerten Realitätsgrad aufweisen, ganz beiläufig auch noch eine E-Kompetenz, die im weiteren Studienverlauf immer mehr zum Tragen kommen wird. Es werden dabei generelle akademische Kernfertigkeiten des wissenschaftlichen Arbeitens ebenso wie Selbst- und Informationskompetenz nachhaltig vermittelt.

\section{Ausblick}

Diese Ausführungen dürfen nicht nur zeigen, wie gelungen sich der E-Bibliotheksschein Anglophone Studien in das Studium eines jeden Studierenden eingliedert oder wie sehr er zu einer noch besseren Verzahnung von Fachbereich und Bibliothek beiträgt, sie dürfen auch gerne Inspiration dafür sein, einen solchen Kursraum für den eigenen Fachbereich zu entwickeln. 


\section{Quellen}

Graf, Dorothee; Borchers, Melanie; Caeners, Stefanie; Hempel, Margit. 2012. E-Bibliotheksschein Anglophone Studien/Studies: Einführung in die fachwissenschaftliche Recherche auf Moodle-Basis. http://www.uni-due.de/ub/schulung/ anglistik_e-schein.

Universität Duisburg-Essen.2011. Prüfungsordnung für den Bachelor-Studiengang mit der Lehramtsoption Gymnasien/Gesamtschulen an der Universität Duisburg-Essen vom 26. August 2011. http://www.uni-due.de/imperia/md/ content/zentralverwaltung/bereinigte_sammlung/8_00_26.pdf.

Universitätsbibliothek der Universität Duisburg-Essen.2010. Bibliotheksschein. http://www.uni-due.de/ub/abisz/schein.shtml.
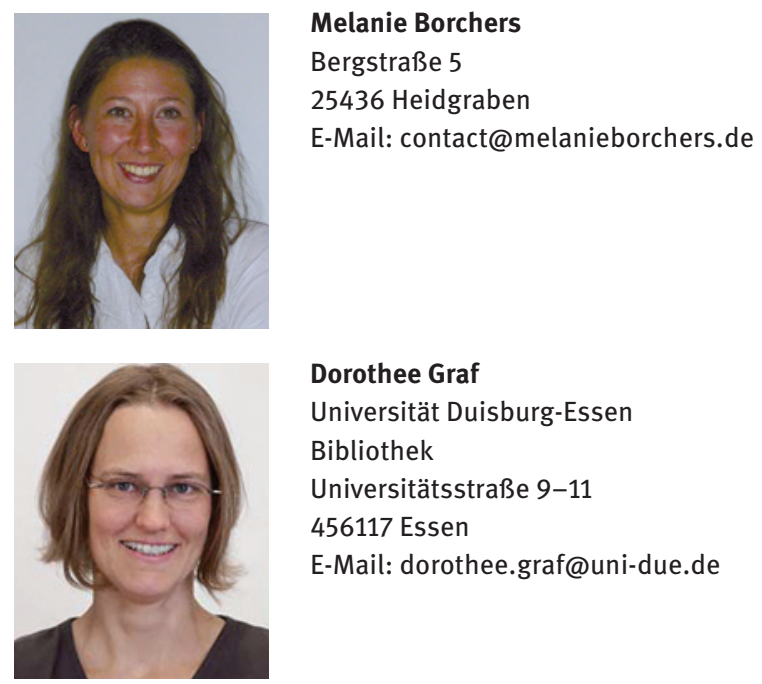

\section{Dorothee Graf}

Universität Duisburg-Essen

Bibliothek

Universitätsstraße 9-11

456117 Essen

E-Mail: dorothee.graf@uni-due.de 


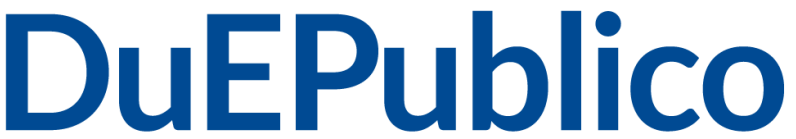

Duisburg-Essen Publications online offen im Denken

$\mathbf{U b} \mid \begin{aligned} & \text { universitäts } \\ & \text { bibliothek }\end{aligned}$

Dieser Text wird über DuEPublico, dem Dokumenten- und Publikationsserver der Universität Duisburg-Essen, zur Verfügung gestellt. Die hier veröffentlichte Version der EPublikation kann von einer eventuell ebenfalls veröffentlichten Verlagsversion abweichen.

DOI: $\quad 10.1515 /$ bd-2014-0125

URN: urn:nbn:de:hbz:464-20201008-105054-2

Alle Rechte vorbehalten.

Dieser Beitrag ist mit Zustimmung des Rechteinhabers aufgrund einer (DFGgeförderten) Allianz- bzw. Nationallizenz frei zugänglich. 